\title{
Parálisis criptogénica del tercer par craneal. Reporte de caso
}

\section{Cryptogenic paralysis of the third cranial pair. Case report}

\author{
Jhony Alejandro Díaz-Vallejo (iD) ${ }^{1}$, José Manuel Bastidas-Acosta (iD) ${ }^{2}$, Luna Valeria Gómez-Bastidas (iD) ${ }^{3}$, \\ Diana Lorena Martínez-Muñoz (iD 4
}

1. Universidad de Caldas. Manizales, Colombia. Correo: alejandrodiazval@gmail.com - https://orcid.org/0000-0002-0784-6688

2. Universidad de Caldas. Manizales, Colombia. Correo: jomabasti@hotmail.com - https://orcid.org/0000-0002-1462-3319

3. Universidad de Caldas. Manizales, Colombia. Correo: luna.521825326@ucaldas.edu.co - https://orcid.org/0000-0002-0228-5068

4. Universidad de Caldas. Manizales, Colombia. Correo: loremar9801@gmail.com - https://orcid.org/0000-0001-5610-2135

Tipología: Reporte de caso clínico

Para citar este artículo: Díaz-Vallejo JA, Bastidas-Acosta JM, Gómez-Bastidas LV, Martínez-Muñoz DL. Parálisis criptogénica del tercer par craneal. Reporte de caso. Duazary. 2021 septiembre; 18(3 número especial): 109-113. Doi: https://doi.org/10.21676/2389783X.4265

Recibido en abril 19 de 2021

Aceptado en junio 29 de 2021

Publicado en línea en agosto 26 de 2021

\section{RESUMEN}

Palabras clave: parálisis del tercer par craneal; pediatría; parálisis oculomotora.
El nervio motor ocular común o tercer par craneal tiene su origen en el núcleo oculomotor en el mesencéfalo. Emerge de allí y se incorpora en la órbita por medio de la fisura orbitaria superior inervando los músculos elevadores del párpado superior, recto superior, recto medial, recto inferior y oblicuo inferior. Su afectación en pediatría es poco frecuente y suele ser ocasionada por causas congénitas, traumáticas, infecciosas, tumorales, vasculares, tóxicas y desmielinizantes. El propósito es reportar e informar sobre caso de paciente con el diagnóstico de una oftalmoplejía aguda dolorosa del tercer par craneal izquierdo. Se presentó el caso de una paciente femenina de 5 años de edad con el diagnóstico de una oftalmoplejía aguda dolorosa del tercer par craneal izquierdo, de etiología desconocida. Todas las pruebas complementarias fueron normales. Fue manejada con rehabilitación ocular y resolución espontanea sin recurrencias. Este nervio puede presentar una parálisis completa o una parálisis parcial o también llamada incompleta, las parálisis pediátricas del nervio oculomotor son trastornos poco frecuentes, con diferentes mecanismos etiológicos, que difieren de los casos en adultos. Su tratamiento y pronóstico dependen de la etiología, los cuales pueden ser variados.

\section{ABSTRACT}

Keywords: Third cranial pair paralysis; Pediatrics; Oculomotor paralysis.
The common ocular motor nerve or third cranial nerve originates from the oculomotor nucleus in the midbrain. It emerges from there and is incorporated into the orbit through the upper orbital fissure, innervating the lifting muscles of the upper eyelid, superior rectus, medial rectus, inferior rectus and inferior oblique. Its affectation in pediatrics is rare and it is usually caused by congenital, traumatic, infectious, tumorous, vascular, toxic and demyelinating causes. The purpose is to report and inform about the case of a patient with the diagnosis of an acute painful ophthalmoplegia of the third left cranial pair. A 5-year-old female patient was presented with the diagnosis of acute painful ophthalmoplegia of the third left cranial pair, of unknown etiology. All complementary tests were normal. She was managed with ocular rehabilitation and spontaneous resolution without recurrences. This nerve can present a complete paralysis or a partial or also called incomplete paralysis. Pediatric oculomotor nerve paralyses are rare disorders, with different etiological mechanisms, which differ from cases in adults. Their treatment and prognosis depend on the etiology, which may be varied. 


\section{INTRODUCCIÓN}

El nervio motor oculomotor o tercer par craneal contiene fibras motoras y parasimpáticas, tiene su origen real en los núcleos somatomotor y núcleo motor accesorio, situados en el pedúnculo cerebral, por delante de la sustancia gris periacueductal en el mesencéfalo ${ }^{1,2}$. Emerge a nivel del espacio interpeduncular (origen aparente), cursa superior y lateral a las apófisis clinoides posteriores e ingresa en la pared lateral del seno cavernoso y se incorpora en la órbita por medio de la fisura orbitaria superior donde se divide en dos ramos, el ramo superior inerva los músculos recto superior y elevador del párpado superior, y el ramo inferior inerva el recto interno, el recto inferior y el oblicuo menor ${ }^{3,4}$.

Este nervio puede presentar una parálisis completa donde se encuentra ptosis palpebral, estrabismo divergente, diplopía y midriasis paralítica; o una parálisis parcial o incompleta donde se afecta en forma aislada algunos músculos oculomotores, sin compromiso de la pupila, de igual pueden ser congénitas 0 adquiridas ${ }^{5,6}$. Las parálisis pediátricas del nervio oculomotor son trastornos poco frecuentes ${ }^{7}$, con diferentes mecanismos etiológicos dividiéndose ya sea por su localización según su recorrido, a nivel del tronco cerebral, espacio subaracnoideo, seno cavernoso o a nivel orbitario; o por sus causas, en congénitas, traumáticas, infecciosas, tumorales, vasculares, tóxicas y desmielinizantes ${ }^{8}$, que difieren de los casos en adultos, donde predominan las causas de aneurismas y microvasculares ${ }^{9,10,11}$.

Teniendo en cuenta lo poco frecuente de la presentación de la parálisis criptogénica del tercer par craneal, su afectación predominante en niños y el pronóstico que puede tener, se decide presentar este reporte de caso clínico con el objetivo de dar a conocer las características clínicas que permiten realizar el diagnóstico de esta afección.

\section{REPORTE DE CASO CLÍNICO}

Paciente femenina de 5 años de edad, en adecuado estado nutricional y neurodesarrollo, sin antecedentes patológicos de importancia ni traumatismos craneoencefálicos, con cuadro clínico de aproximadamente 6 días de evolución, el cual inicia de forma súbita con dolor en el ojo izquierdo, asociado a fiebre, su madre nota caída del parpado superior izquierdo por lo cual deciden consultar.

Los hallazgos al examen físico se encuentra, pstosis palpebral izquierda, exotropia izquierda (figura 1), agudeza visual cercana en ojo derecho (OD) 20:20, ojo izquierdo (OI) 20:20, reflejos fotomotor y consensual ambos ojos 2+, pupilas en luz OD 3.0 milímetros $(\mathrm{mm})$, OI $4.0 \mathrm{~mm}$, en oscuridad OD 3.5 $\mathrm{mm}$, Ol $0.0 \mathrm{~mm}$, limitación a la aducción OI 2+, limitación a la supra e infraducción OI 2+; En ambos ojos cornea clara, cámara anterior formada, iris normal, cristalino transparente, fondo de ojo en ambos ojos con nervio óptico definido, anillo neurorretiniano rosado, excavación/papila de 0.2 , macula sana, vasos normales, afebril e hidratada. Ojo derecho sano y el resto de examen físico y neurológico normal.

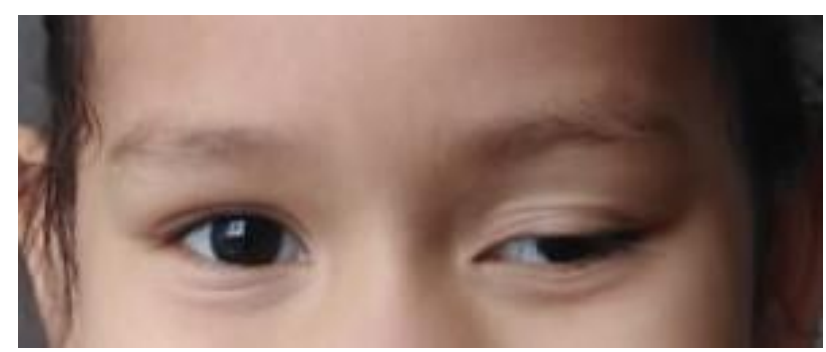

Figura 1. Ptosis palpebral izquierda con mirada desviada a la izquierda.

En cuanto a los exámenes complementarios, se le realiza hemograma con hemoglobina: $13.2 \mathrm{~g} / \mathrm{L}$, hematocrito: $39 \%$, leucocitos: $6400 / \mathrm{mm} 3$, neutrófilos: 4900/mm3, linfocitos: $950 / \mathrm{mm} 3$, monocitos: $310 / \mathrm{mm} 3$, plaquetas: 202/mm3, glicemia: $72 \mathrm{mg} / \mathrm{dL}$. Tomografía axial computarizada y resonancia magnética simple y contrastada sin imágenes compatibles con masas, isquemias, abscesos, aneurisma o hidrocefalia, sin embargo, se presenta como hallazgo incidental un quiste aracnoideo retrocerebeloso.

Manejada con terapias ortopticas, parche ocular alternante 5 horas al día. El dolor ocular se mantiene durante otros pocos días. A partir de los 8 días del inicio del cuadro clínico se constata mejoría de los 
movimientos, la ptosis palpebral se mantiene sin variación, así como la no reactividad lumínica. Se le indica seguimiento mensual con neuro oftalmología pediátrica y alta hospitalaria.

\section{DISCUSIÓN}

Se denomina parálisis criotogenica del III par craneal, cuando la causa de la lesión no es identificable ${ }^{12}$. Ptosis palpebral, parálisis de los movimientos oculares como: elevación, descenso y aducción y no contracción del esfínter pupilar, son características en la lesión de este par craneal ${ }^{13}$. En la paciente se evidencia una parálisis del tercer par craneal mono ocular izquierda, sin compromiso derecho y sin otros hallazgos físicos. A pesar de un constante seguimiento no se logra encontrar una causa clara.

En niños, las causas infecciosas son las más usuales ${ }^{14}$, aunque pueden ser difíciles de detectar, debido a la frecuencia de episodios infecciosos agudos en ellos, con frecuencia son poco sintomáticos y no es fácil realizar pruebas para su detección.

Las pruebas de neuroimagen son importantes en el diagnóstico de esta patología, y es de elección la RM puesto que permite definir la anatomía en su trayecto ${ }^{15-17}$. Las pruebas de neuroimagen realizadas a nuestra paciente son normales, al igual que las de laboratorio con el hemograma, y sin antecedentes importantes en la paciente, no se puede aclarar la etiología a la paciente.

Se propone una clasificación clínica de severidad (Tabla 1) en la cual se estima el grado de compromiso y de acuerdo a ésta el tiempo y la posibilidad de recuperación que se pueda dar ${ }^{18}$. La paciente se clasifica en grado III, dónde según los estudios de Dimopoulos, se espera una resolución completa dentro de las 4 a 8 semanas siguientes.
Tabla 1: Escala de severidad de parálisis del III par craneal.

\begin{tabular}{|c|l|l|}
\hline Grado de ptosis & $\begin{array}{l}\text { Ausencia de } \\
\text { ptosis }\end{array}$ & 0 \\
\cline { 2 - 3 } & Ptosis parcial & 1 \\
\cline { 2 - 3 } & $\begin{array}{l}\text { Ptosis } \\
\text { completa }\end{array}$ & 2 \\
\hline $\begin{array}{c}\text { Paresia músculos } \\
\text { extra oculares }\end{array}$ & Ausente & 0 \\
\cline { 2 - 3 } Reacción pupilar & Presente & 1 \\
\hline & Presente & 0 \\
\cline { 2 - 3 } & Ausente & 1 \\
\hline
\end{tabular}

Fuente: Elaboración propia basada en Dimopoulos ${ }^{18}$.

Tabla 2: Grados de severidad de parálisis del III par craneal.

\begin{tabular}{|l|l|}
\hline Grado I & 1 punto \\
\hline Grado II & 2 puntos \\
\hline Grado III & 3 puntos \\
\hline Grado IV & 4 puntos \\
\hline
\end{tabular}

Fuente: Elaboración propia basada en Dimopoulos ${ }^{18}$

Se ha visto que en algunos casos puede haber con el tiempo resolución completa espontáneamente ${ }^{5}$, sin embargo, en su manejo se debe tener en cuenta múltiples factores, incluyendo la edad del paciente, la historia clínica, el nervio específico afectado, los hallazgos del examen físico y los diagnósticos médicos coexistentes ${ }^{19}$, aunque también de su etiología en las cuales se puede brindar desde rehabilitación oculomotora hasta manejo quirúrgico según sea el caso ${ }^{20,21}$.

$\mathrm{Su}$ pronóstico depende de tres factores importantes, el comienzo de la parálisis, el tiempo transcurrido entre el comienzo de la parálisis y la intervención, y el tipo de intervención que se realice ${ }^{22}$.

Se concluye que este nervio puede presentar una parálisis completa o una parálisis parcial o también llamada incompleta; las parálisis pediátricas del nervio oculomotor como la presentada en la paciente, son trastornos poco frecuentes, con diferentes mecanismos etiológicos, que difieren de los casos en adultos. Su tratamiento y pronóstico dependen de la etiología, los cuales pueden ser variados. 


\section{DECLARACIÓN SOBRE CONFLICTOS DE INTERÉS}

Declaramos no tener ningún conflicto de intereses.

\section{CONTRIBUCIÓN DE LOS AUTORES}

Primer autor: Trabajo de campo y redacción. Segundo autor: Trabajo de campo y redacción. Tercer autor: Trabajo de campo y redacción. Cuarto autor: Trabajo de campo y redacción.

\section{REFERENCIAS BIBLIOGRÁFICAS}

1. Saraux H, Lemasson C, Offret GH. Renard, Anatomía e Histología del Ojo. Barcelona: Ed. Masson; 1985:313-320

2. Glaser JS, Neuro oftalmología. Barcelona: Ed. Salvat; 1982. 247:251-252

3. Moses RA. Fisiología del Ojo. Buenos Aires : Ed. Panamericana;1980:312-314

4. Pró EA. Anatomía Clínica, 2 Edición. Buenos Aires: Panamericana; 2014:292.

5. Saucedo Salcedo S. Parálisis del III par craneal Presentación de un caso y revisión de la literatura. Lux Médica. 2009; 4(11):45-53. Disponible en: https://revistas.uaa.mx/index.php/luxmedica/articl e/view/1693

6. Prawda Witemberg M, Lozano Pratt A. Parálisis congénita incompleta del tercer par cran. México: Ciencias Médicas; 2002. Disponible en: https://pesquisa.bvsalud.org/portal/resource/pt/lil $-124660$

7. Fuentes Pelier D, Hodelín Tablada R, Cruz Egued K. Parálisis criptogénica del III par craneal. Tratamiento con corticosteroides. Rev Neurol. 2000;31:800. Disponible en: https://www.neurologia.com/articulo/2000353

8. Pozo Alonso AJ, Pozo Lauzán DR, Hernández Meilán M, Sayú Stewart JM. Parálisis criptogénica del III par craneal. Rev Cubana Pediatr. 2013 Jun; 85(2): 252-257. Disponible en: http://scielo.sld.cu/scielo.php?script=sci_arttext\&p id=S0034-75312013000200012

9. Mudgil AV, Repka MX. Ophthalmologic outcome after third cranial nerve palsy or paresis in childhood. J Am Assoc Pediatr Ophthalmol Strabismus JAAPOS. 1 de febrero de 1999;3(1):2-8. Disponible en: https://pubmed.ncbi.nlm.nih.gov/10071894/

10. Tamhankar MA, Biousse V, Ying GS, et al. Isolated third, fourth, and sixth cranial nerve palsies from presumed microvascular versus other causes: a prospective study. Ophthalmology. 2013;120(11):2264-2269. Disponible en: http://europepmc.org/article/PMC/3795864

11. Guy JR, Day AL. Intracranial aneurysms with superior division paresis of the oculomotor nerve. Ophthalmology. 1989;96(7):1071-1076. Disponible en:

https://www.scopus.com/record/display.uri?eid=2s2.0-

0024322091\&origin=inward\&txGid=89c138a4622d cfbcd93a2e7fbb7e8661

12. Jiménez Montañez $L$, Martínez Fernández $M$, Elizalde Usechi M, López Pisón J. Uso de corticosteroides en la parálisis criptogénica del III par craneal. A propósito de un caso. Rev Neurol. 1999;29:1052-4. Disponible en: https://www.neurologia.com/articulo/98913

13. Cabarga Haro CE, Gonzáles Mesa MI, Legrá Nápoles S, Velázquez MA. Parálisis del tercer par. Presentación clínica. Revista electrónica de portales médicos. 2009. Disponible en: https://www.portalesmedicos.com/publicaciones/ articles/1453/1/Paralisis-del-Tercer-par-

Presentacion-Clinica.html

14. Chan C, Sugg RL, Steinman L. Isolated oculomotor palsy after measles immunization. Am J Ophthalmol. 1980;89:446-8. Disponible en: https://www.ajo.com/article/0002-9394(80)90019$7 / p d f$

15. Olivares Fernández V, Álvarez-Linera J, TorresAlcázar A, Martínez Salcedo E, Alarcón-Martínez H, 
Casas Fernández C. Neuritis del III par: diagnóstico por imagen. Rev Neurol. 2012;54:59. Disponible en: https://www.neurologia.com/articulo/2011641

16. Chou KL, Galetta SL, Liu GT, et al. Acute ocular motor mononeuropathies: prospective study of the roles of neuroimaging and clinical assessment. J Neurol Sci. 2004;219(1-2):35-39. Disponible en: https://www.scholars.northwestern.edu/en/public ations/acute-ocular-motor-mononeuropathies-

prospective-study-of-the-role

17. Jacobson DM, Trobe JD. The emerging role of magnetic resonance angiography in the management of patients with third cranial nerve palsy. Am J Ophthalmol. 1999;128(1):94-96. Disponible en: https://www.ajo.com/article/S00029394(99)00107-5/fulltext

18. Dimopoulos VG, Fountas $\mathrm{KN}$, Feltes $\mathrm{CH}$, Robinson JS, Grigorian AA. Literature review regarding the methodology of assessing third nerve paresis associated with no ruptured posterior communicating artery aneurisms. Neurosurg Rev. 2005; 28(4): 256-60. Disponible en: https://link.springer.com/article/10.1007/s10143005-0393-6
19. Pineles SL, Velez FG. Isolated Ocular Motor Nerve Palsies.J Binocul Vis Ocul Motil. 2018;68(3):70-77. Disponible en: https://pubmed.ncbi.nlm.nih.gov/30196784/

20. Scheiman M, Wick B. Tratamiento Clínico de la Visión Binocular Disfunciones heteroforicas, acomodativas y Oculomotoras. Madrid: Ed. Ciagami; 1996:97-98

21. Roy F. Terapéutica Oftalmológica. Buenos aires: Ed. Medica Panamericana; 1983:498-499.

22. Hamer J. Prognosis of oculomotor palsy in patients with aneurysms of the posterior communicating artery. Acta Neurochir 1982 66: 173$185 . \quad$ Disponible en: https://link.springer.com/article/10.1007/BF02074 504 